\title{
Torque and Capacity Measurement in Rotating Transmission
}

\author{
Ivan Grabar ${ }^{1}$, Igor Korobiichuk ${ }^{2([)]}$, and Oleg Petruk ${ }^{2}$ \\ ] Zhytomyr National University of Agriculture and Ecology, Zhytomyr, Ukraine \\ i van-grabargukr. net \\ 2 Industrial Research Institute for Automation and Measurements PIAP, \\ Warsaw, Poland \\ \{iko robi ichuk, opetruk\}@piap. pl
}

\begin{abstract}
The task of measuring, monitoring and recording of the torque, mechanical stress and capacity is relevant for most transmissions of the process and transport machines. The importance of this problem is increased by an order in terms of unsteady loads (random, impulsive, alternating, etc.). The disadvantage of its cortect solution by experimental methods is substantially limited by the need for expensive and unreliable, environmentally dangerous current collectors. To measure the dynamic and kinematic parameters of the rotating transmission by modern information computer technologies, we propose equipping the mechanical transmission with two additional half-couplings with point magnets fixed thereon, the scope of each includes one or more Hall sensors. The application of the developed technique allows determining the torsional rigidity of the transmission experimentally, and allows recorting the actual torque and capacity per turn (or a part of the turn) of the transmission of a process or transport machine and record the same online in the computer electronic memory. This, in turn, allows an online assessment of the residual transmission resource, machine operation per shift and generate its operation history, which provides an invaluable information both to the machine developer and owner.
\end{abstract}

Keywords: Mechanical transmission - Tachometer - Measurement systems Torque $\cdot$ Capacity $\cdot$ Rotating transmission

\section{Introduction}

The task of measurement, control and registration of torque, mechanical stresses and power occurs in most cases of transmissions of technological machine and transporters. The importance of this problem is increasing in the conditions of non-stationary loads (random, impulse, alternating, etc.). The tensometric methods are often applied to increase the accuracy of the correct solution to this task. It will be shown later, that their application is significantly reduced with the necessity to use the expensive and fault-tolerant methods of the transmission of the signal from rotor to stator. Moreover, these signals are not reliable. At the same time, the application of the perfect means of technological process parameter control is the main task of the production automation. The conirol of the angular speed, and especially, the torque and capacity, allows 
preventing the technological machinery overloading. It also makes possible to register the real history of their operation. The given systems obtain the special significance at drive unit and transmission tests, at their maximum load bearing capacity research, at the investigation of the dynamic parameters and transmission efficiency, gear boxes, reducers, fans, pumps. The bolted connection assemley automation, the crank connection rod and crank rocker mechanisms and transporters operation also require the experimental measurement $(\Omega \mathrm{MN})$.

\section{The Current State of the Problem}

Nowadays the market offers a great variety of experimental means for measuring the angular velocity, lorque and the capacity on the transmissions which rotate [1-17]. Such methods as: the method of measuring of the centrifugal force which is proportional to the square of the angular velocity; the induction and magnet induction methods, stroboscopic method and others have gained a good reputation arnong those which have been studied. The laser portative folotachometers of the types AKIP-9201 and AKIP-9202 [14] demonstrated their bests at the application. They allow determining the angular velocities by non-contact method; possess the comfortable system of the laser sighting on the object which is being investigated. The given tachometers are capable of providing the effective measurements of rotating shafts of energy for manufactory equipment, as well as for household appliances. Besides, they are comfortable to be applied to the scientific researches. It is important to mention, that the given fototachometers function in the range of $100000 \mathrm{RPM}$.

The modern means of the angular velocity measurement are also offered by automobile manufacturers, especially for ABS system [15]. Figure 1 shows the example of such tachometer, where 1 - is the permanent magnet, 2 -is the trunk; 3 -is the winding; $4-$ is the iron circuit; 5 - is the pulse drive.

The given system is characterized by the high precision for non-stationary processes. It is also able to measure the modes with great values of angular accelerations for both signs.

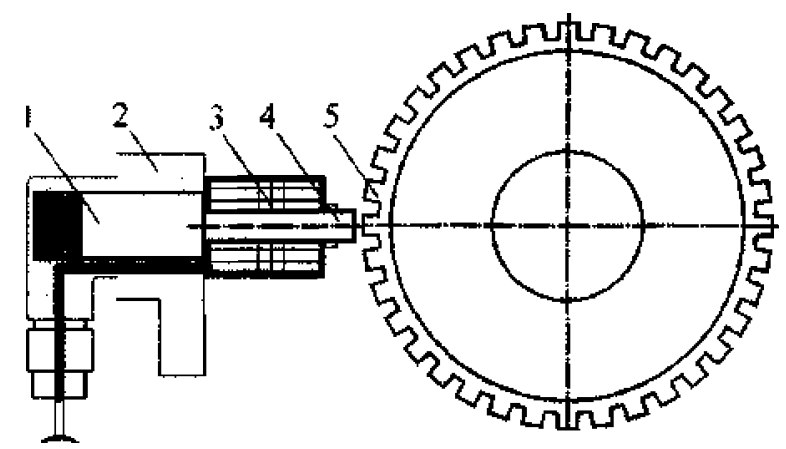

Fig. 1. Construction of tachometer 
The modern systems of torque measurement are offered by the famous German companies such as HBM, Burster, Japanese company Kyowa and Korean firm Dacell. and also by other world leaders in mechatronics and automation. The market offers a great variety of dynamometers of torque which are based on the tensometry application. i.e. the converting shaft mechanical deformations into electric signal and signaling the computer by contact or non-contact way. Figure 2 demonstrates the samples of torque dynamometer.

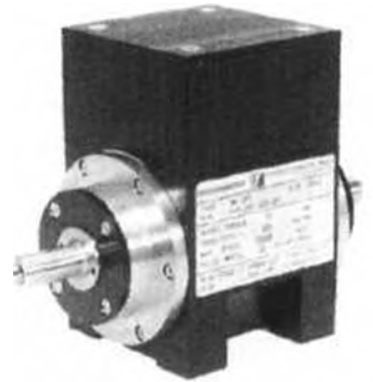

a)

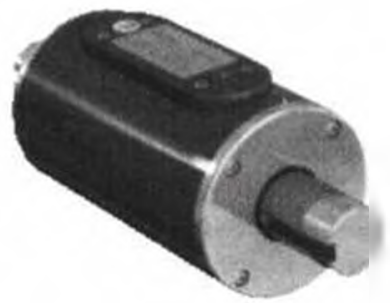

b)

Fig. 2. The torque dynamometers [16, 17]

\section{Task Formulation}

[10] describes the system of non-contact sensor of angular velocity and torque with the transmission of information from dynamometer rotor to stator. The tenso-system is attached to the sensor of the dynamometer. The system includes four tenso-sensors linked by bridge circuit and it is connected to the power supply system. The strain gauge bridge supply is misbalanced by torque. Here the misbalance signal is proportional to the value of torque. The misbalance signal is transmitted to the transmitter signal by the tenso-amplifier and is sent to stator receiver. The receiver is placed on the rotor. The given system is widely used in the modern systems of the measurement of torque on the shaft of the rotating transmission. It possesses huge rigity and it means that it possesses the high values of critical rotations. These rotations are characterized by high susceptibility and speed and they can be used at any form of a torque feature depending on time (Fig. 3).

The drawbacks of the given system are: the complexity. high cost, the necessity to break the kinematic scheme in order to assembly the dynamometer. The low fault tolerance at the transmission of signal from dynamometer rotor to stator using optical, radio or magnet and induction channel is another disadvantage of the system. The contact methods of the transmission of signal from rotor to stator have many more limitations. Neither noble metals (silver, gold), nor graphite or mercury moving contacts are able to provide the signal transmission from the rotor of dynamometer to the stator at high revolutions (more than $100 \mathrm{RPM}$ ). It is impossible to achieve without some modifications which can be caused by the intermittent signals, sparking, and 


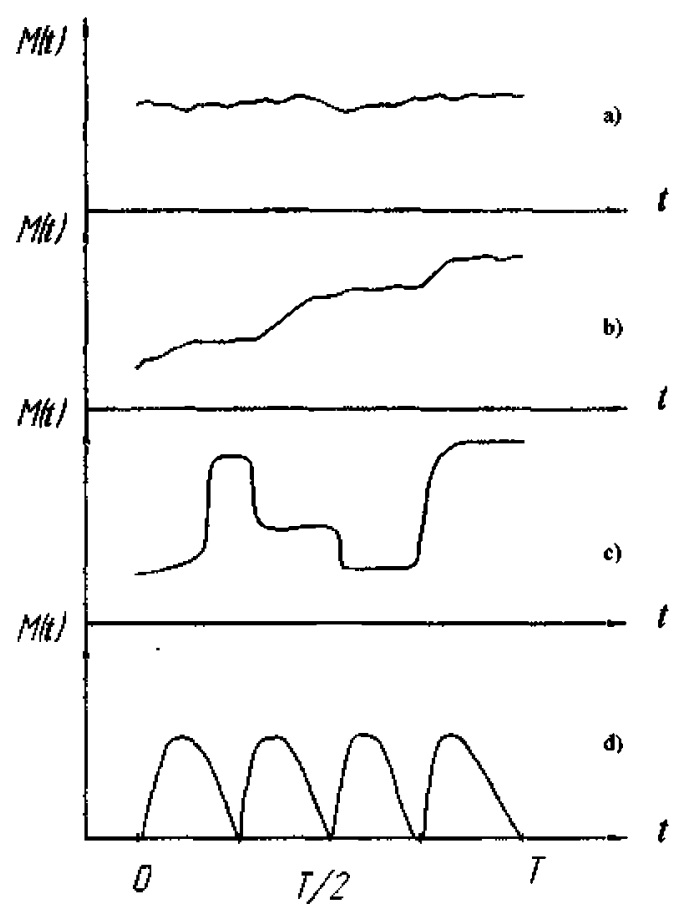

Fig. 3. The typical dependencies of torque change of transmission shaft for its single rotation

mercury viscosity. The application of the supersensitive percolation-fractal sensors which can replace the tenso-amplifiers $[3,12]$ does not change the situation. It has been shown [1], that the great breakthrough in tensometry elimination (both contact and non-contact) is possible to achieve by denying using it at all. The signal about the value of torque is formed by the system of the direct measurement of the deformation of the shaft sensor without converting it into electric signal of tensometric bridge. The given system has demonstrated a lot of advantages at torque measurement, angular velocity and capacity for transmissions which are characterized by great values of twist angle (long shafts) and little revolutions (up to $300 \mathrm{RPM}$ ). The optical systems (Jaser radiator and optrone receivers), induction systems and Hall sensors were used to obtain the non-contact measurement of the twist angle.

\section{The Research Results}

In order to find the partial solution to the problem of rotating transmission functioning in the wide range of operating modes, it is recommended to apply the DynamometerTachometer-Watt-meter by Professor Grabar [2].

We suggest equipping the mechanical transmission by two half-couplings with the fixed spot magnets on them. A Hall sensor D1-D2 (Fig. 4) is placed on the coverage of each magnet. It is performed to measure the dynamic and kinematic parameters of the 
rotating transmission applying the means of modern information-computer technologies.

If the torque $M$ is active in the shaft section, this changes the phase angle between spot magnets 1 and 2 by the value $\Delta \varphi$.

Then, the transmission turn angle $\varphi=\omega t$ and $\Delta \varphi=\omega \Delta \tau$.

Hence: $\omega=\frac{2 \pi}{\tau}$. Then, the torque on the shaft:

$$
M=C \cdot \Delta \phi=C \frac{2 \pi}{\tau} \Delta \tau
$$

And, correspondingly, the capacity:

$$
N=M \cdot \omega=C\left(\frac{2 \pi}{\tau}\right)^{2} \Delta \tau
$$
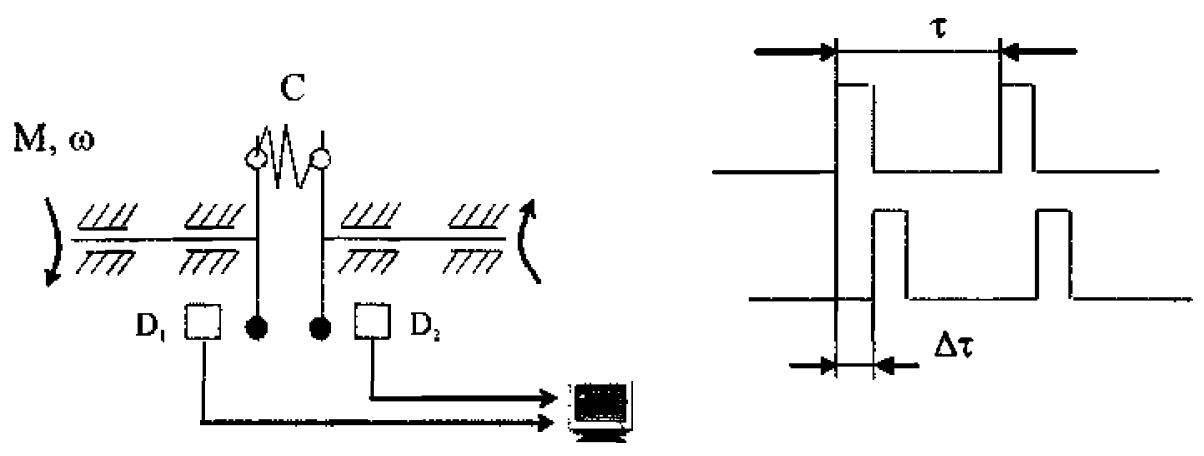

Fig. 4. Construction of measurement systems

Therefore, we obtain the opportunity to register the real torque and the capacity of every single revolution (or a part of a revolution) of any technological machine or transporter. It is done by measuring $\tau$ and $\Delta \tau$ only, and by the experimental determination of the transmission torsional rigity $\mathbf{C}$ in the section D1-D2. It allows online registering of these processes and storing them in the computer's memory. In its turn, it makes possible to provide online estimation of the remaining transmission resource, the operation of machines per shift and to form the history of its operation. This provides both the developer and the owner of the machine with the valuable information.

\section{The Experimental Research}

The testing of the offered system efficiency to measure the angular velocity, torque and the rotation transmission efficiency was experimentally conducted with two-rotor timber grinder (Fig. 5). 


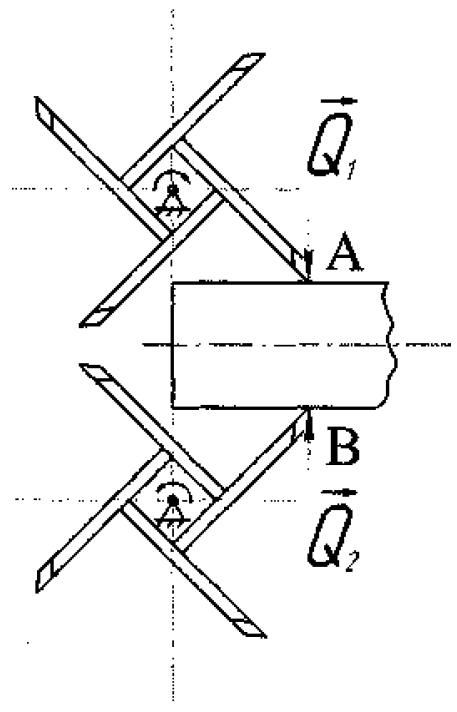

Fig. 5. The scheme of two-rotor timber grinder

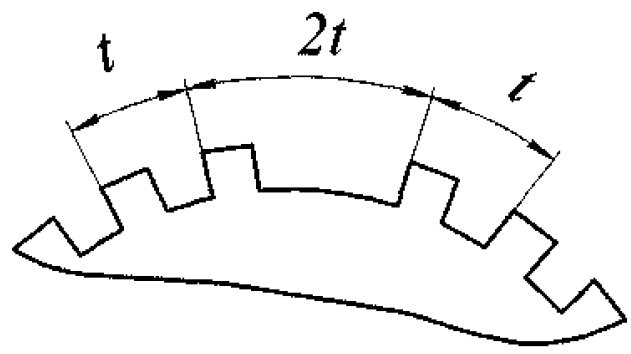

Fig. 6. The timing drive marker area

The rotor shaft torque mathematic model is designed to be used to grind any cross section timber:

$$
M_{R(i)}=Q_{R(i)} R_{j p} \sin \left(x \frac{n-i}{n}\right)
$$

where: $Q_{R i}=\left(S_{s e g(i)}-S_{s e g(i-1)}\right) \tau_{z p}$.

In particular, for the cylindric timber:

$$
S_{s e g(i)}=R_{b p}\left(\beta_{i}-\frac{1}{2} \sin 2 \beta_{i}\right)
$$




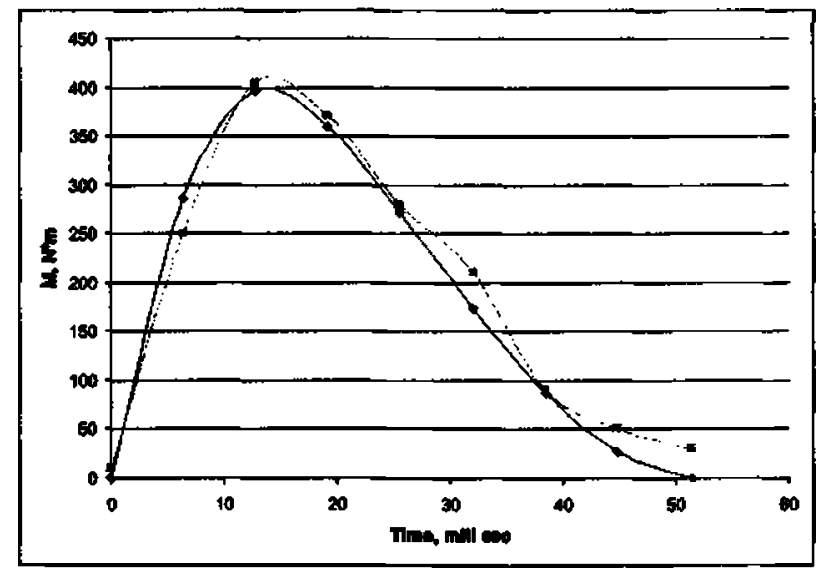

Fig. 7. The theoretical (blue) and the experimental (red) dependencies of the torque on the shaft of rotor timber grinder. The pine timber of the cylindrical form by the diameter $100 \mathrm{~mm}$ and voltage cutoff $9.2 \mathrm{MPa}$ (Color figure online)

$n-$ is the number of measurements per one operation cycle (in the given research

$n=9, Z=36$ ),

$R_{f p}, R_{b p}-$ is the milling cutter radius and the timber radius.

Two timing drives were placed on the shaft butts Fig. 1. One notch was distracted from every drive in order to identify the signals (Fig. 6) is the marker area (Fig. 7).

\section{The Propositions as to the Application}

The proposed system of transmission parameter measurement has some important advantages. Firstly, it is not necessary to change the scheme, to break the kinematic scheme and to find some extra space to assemble the additional elements of dynamometers. Moreover, every, among the mentioned machines, requires the essential re-equipping in order to attach the torque dynamometers. Such measurements are easy to conduct on the special stands. But, it is not always possible to reproduce the real operating values of the loading modes with such stands.

Some variants of technical solutions are offered applying our methodology:

Variant A. The long shaft ( $/ \mathrm{D}>50$ ), low revolutions $(n<300 \mathrm{RPM})$ are the transmissions of stone processing and agricultural machines, screw transporters, mixers, etc. The measurement system ( $\Omega \mathrm{M} \mathrm{N}$ ) consists of two spot magnets (for example, of the cylindrical form and the diameter 1-2 mm and 1-3 mm high), two Hall sensors, two screen lines with USB ports to transmit the impulse signals to the computer to be registered, processed and stored. If it is needed, the spot magnets can be placed not on the surface of the shaft, but on the extra weight or special disk. It allows increasing the radius of the trajectory of the magnet movement and, correspondingly, providing the precision of the torque measurement. It has been shown, that the mentioned above 
lypes of machines allow finding some space $1-2 \mathrm{~cm}^{3}$ and the space to assembly the screen lines by the diameter $4 \mathrm{~mm}$ and $5-10 \mathrm{~m}$ long. It is possible at the whole layout density and without any extra changes of the design.

Variant B. A shaft of any length, the low revolutions ( $<100$ RPM) are the transmissions for either of variant $A$ machines, or the machines used to mix building mixtures, grinding and distribution of feed, fertilization and others. The measurement system ( $\Omega \mathrm{MN}$ ) consists of (as in previous variant) two spot magnets, two Hall sensors, two screen lines with USB ports to transmit impulse signals to the computer to be registered, processed and stored. It is also used to provide the assembly of the element of the low torsional rigity (special elastic coupling - Fig. 4). It localizes and significantly (in 10-100 times) rises the increased torque value between the left and right butt of the shift.

Variant C. If the variant B does not provide the possibility to assembly the low torsion rigity element, but, there is the access to the shaft butts, then the set drives are attached to the shaft butts (Fig. 1) made of ferromagnetic material and the number of notches $Z$, selected from the condition $Z=\frac{2 \pi}{4 \Delta \phi}$.

This variant practically eliminates the limits for the revolution velocity dependence of torque (t) and is universal one to be applied.

\section{Conclusions}

1. The methodology for the measurement of the angular velocity, torque and capacity $(\Omega \mathrm{M} \mathrm{N})$ on rotating transmission is implemented for the modern systems of technological processes. It can be assembled directly in the technological machine without crucial changes. The set precision enables to measure the mentioned transmission parameters.

2. It has been shown, that to implement the process of the parameter $(\Omega \mathrm{MN})$ registration it is recommended to measure only three parameters: $\tau, \Delta t$ and $C$ which is the period between impulses, shaft deformation impulse shift and the active transmission part torsion rigity.

\section{References}

1. Kuo, B.C., Golnaraghi. F.: Automatic Control Systems, 609 p.Wiley, New York (2002)

2. Grabar, I.G.: Patents of Ukraine, No 83475, 85252, 73063, 91664

3. Yahno, O.M.. Grabar, I.G., Taurit, T.G.: Wind Power: the Design and Calculation, 255 p. (2003). Zhitomis

4. Grabar, 1.G., Bezvesilna, O.M., Kubrak, Y.O.: Precolation tensoconvertor of the linear deformation and the theoretical backgrounds of its operation. ZSTU Messenger. Tech. Sci. 1 (46) (2008). №3

5. Avnutov, V.V.: The angular velocity determination by means of circuit. NUNU KPI Messenger. Issue Instr. 28-32 (2014). No. 47 
6. Korobiichuk, I.: Mathematical model of precision sensor for an automatic weapons stabilizer system. Measurement 89, 151-158 (2016). doi:10.1016/j.measurement.2016.04.017147

7. Kravchenko, N.A., Yashkov, V.A., Soldatkin, V.M., Porunov, A.A.: Two-component Flapper Nozzle Transducer of the Angular Velocity, patent RU No 2462723

8. http://www.elec.ru/news/2008/12/25/sovremennye-sredstva-izmerenij-akip-novye-cifrovye. himl. (Tachometers AKIP-9201, AKIP-9202)

9. Yakovlev, D.P.: The device for the torque measurement on the jotating shaft. The papers of Odessa Polytechnic University 1(19) (2003)

10. Krimmel, V.: http:/www.lorenz-m.ru/index.php. The Development and the Future of Torque

11. Gurinov, A.C., Dudnik, V.V., Gaponov, V.L., Kalashnikov, V.V.: htp://ivdon.ru/magazine/ archive/n2y2012/798. Don Engineering Messenger. №2 (2012). The torqne Measurement on Rotating Shafts

12. Popov. A.P., Vinokurov, M.R., Moiseenko, A.A.: Microprocessor System of Non-Contact Control and Torque Measurement. DGU Messenger T.10. №2(45) 243-248 (2010)

13. Grabar, I.G., Grabar, O.I. Gutnichenko, O.A., Kubrak. Y.O.: Percolation-Fracral Materials, Monograph, 370 p. (2007). Zhitomir

14. Petrosyan, D.P., Grigoryan, S.M., Mikalean, S.E.: The comparative estimation of the known methods and the development of the optical method of rotating shaft dynamic parameter measurement. Agriculture Sci. News 9(4), 1-5 (2011)

15. Korobiichuk, l., Bezvesilna, O., Ilchenko, A., Shadura, V., Nowicki, M., Szewczyk, R.: A mathematical model of the thermo-anemometric flowmeter. Sensors 15, 22899-22913 (2015). doi: $10.3390 / \mathrm{s} 150922899$

16. http://m.ustroistvo-avtomobilya.ru/

17. http:/www,prom-tex.org/products/krmom.html 